\title{
The Anisotropy in the Galaxy Velocity Field originated from the Gravitational Pancaking Effect
}

\author{
Yookyung Noh and Jounghun Lee \\ Department of Physics and Astronomy, FPRD, Seoul National University, Seoul 151-747, \\ Korea \\ ykyung@astro.snu.ac.kr, jounghun@astro.snu.ac.kr
}

\begin{abstract}
We analyze the Millennium run semi-analytic galaxy catalog to explore quantitatively the gravitational pancaking effect on the orientation of galaxy velocity field. We first calculate the probability density distribution of the cosine of the angle between the velocity of a field galaxy and the direction normal to a local pancake plane which is determined using two nearest neighbor field galaxies. A clear signal of alignment is detected for the case that the pancake scale is in the range of $5-8 h^{-1} \mathrm{Mpc}$. The tendency of the velocity-pancake alignment is found to still exist when the pancakes are determined using three neighbor galaxies, indicating that it has a spatial coherence. The degree of the velocity-pancake alignment is shown to increase with the velocity magnitude and the local density, while it decreases with the separation distance from the galaxy to the pancake and disappears when the pancake has a filamentary shape. A final conclusion is that our work may provide another clue to understanding the large-scale structure in the universe.
\end{abstract}

Subject headings: cosmology:theory - large-scale structure of universe

It is an undeniable fact that there are large-scale anisotropies in the universe. In the context of the cold dark matter (CDM) paradigm, the large-scale anisotropies are understood as a nonlinear manifestation of the primordial tidal field (Bond, Kofman, \& Pogosyan 1996).

The large-scale anisotropy induced in the distribution and the orientation of galaxies has been extensively studied both observationally and theoretically (Flin \& Godlowski 1986; Barnes \& Efstathiou 1987; Garrido et al. 1993; Lee 2004; Navarro et. al. 2004; Trujillo et. al. 2005). But, its presence in the galaxy velocity field has not attracted enough attention so far due to the difficulty in measuring accurately the galaxy velocities from observations. 
The standard theory of structure formation based on the CDM paradigm, however, makes a unique prediction for the anisotropy in the galaxy velocity field. The large-scale coherence in the initial tidal field leads to the formation of two-dimensional sheet-like objects, pancakes (Shandarin et al. 1995; Pauls \& Melott 1995; Bond, Kofman, \& Pogosyan 1996). It will in turn lead the galaxy velocity field to possess planar symmetry, which is referred to as the gravitational pancaking effect.

The pancakes are in fact characteristic of the Zel'dovich approximation (Zel'dovich 1970), according to which the trajectory of a cosmic particle in the comoving coordinate is given as

$$
\mathbf{x}=\mathbf{q}-D(t) \nabla \Psi(\mathbf{q})
$$

Here, $\mathbf{q}$ and $\mathbf{x}$ are the Lagrangian and the Eulerian coordinates, respectively, $D(t)$ is the linear density growth factor, and $\Psi(\mathbf{q})$ is the linear velocity potential. This Zel'dovich approximation breaks down after the moment of the first caustics corresponding to the formation of pancakes. The limitation of the Zel'dovich approximation may be overcome by truncating the nonlinear scale powers, which amounts to smoothing the velocity potential on the galaxy scale (Pauls \& Melott 1995) . Equation (1) thereby is valid for describing the dynamical path of a galaxy till the moment of the pancake formation.

Taking the time derivative of equation (1) and Taylor-expanding $\Psi(\mathbf{q})$ to first order, we have

$$
\dot{x}_{i} \approx-\dot{D} \partial_{i} \Psi(\mathbf{q}) \propto q_{j} T_{i j}, \quad T_{i j} \equiv \partial_{i} \partial_{j} \Psi .
$$

where $T_{i j}$ is the gravitational tidal shear tensor. Since the gravitational collapse to form a pancake occurs along the major principal axis of the tidal shear tensor which is in the direction normal to the plane of the pancake, equation (2) predicts the alignment between $\mathbf{v}$ and the direction normal to the plane of the pancake.

We test this analytic prediction of the Zel'dovich approximation against N-body simulations by analyzing the galaxy catalog from the Millennium run simulation for the concordance $\Lambda$ CDM cosmogony (Springel et al 2005). The catalog contains total 9925229 galaxies in a periodic box of size $500 h^{-1} \mathrm{Mpc}$ with information on various galaxy properties such as position, velocity, mass, and so on. For our purpose, only field galaxies are chosen from the catalog, since for the cluster galaxies the following contaminations may occur in reality: First, for the cluster galaxies the dominant gravitational force would degrade the pancaking effect. Second, if cluster galaxies were contained within spherical virialized objects with tangential velocity dispersion larger than the radial one, the same kind of anisotropy would be misinterpreted as a sign of the pancaking effect.

Total 854738 field galaxies are identified from the catalog according to the criterion 
suggested by El-Ad \& Piran (1997) and Hoyle \& Vogeley (2002) that a field galaxy should have less than three neighbors within a spherical radius of $d+3 \sigma_{d} / 2$ ( $d$ is the mean distance to the third nearest neighbor and $\sigma_{d}$ is the standard deviation on $d$ ).

To examine the velocity-pancake alignment, it is necessary first to identify pancakes from the catalog. Since there is no well-defined pancake-identification method, we propose the following expedient scheme: For each field galaxy, we find two nearest neighbor field galaxies and define a local pancake plane encompassing the field galaxy and the two neighbors.

Let $\mathbf{R}_{1}$ and $\mathbf{R}_{2}$ represent the displacement vectors to the first and the second nearest neighbors from the position of a given field galaxy, respectively, and let $R_{c}$ represent a distance lower-limit satisfying $R_{c} \leq R_{1}<R_{2}$ where $R_{1} \equiv\left|\mathbf{R}_{1}\right|$ and $R_{2} \equiv\left|\mathbf{R}_{2}\right|$. We measure the cosine of the relative angle, $\theta$, between the galaxy velocity vector, $\mathbf{v}$, and the direction normal to the pancake plane as

$$
\cos \theta \equiv \frac{\left|\mathbf{v} \cdot\left(\mathbf{R}_{1} \times \mathbf{R}_{2}\right)\right|}{|\mathbf{v}|\left|\mathbf{R}_{1} \times \mathbf{R}_{2}\right|},
$$

and determine the probability distribution, $p(\cos \theta)$, statistically. We repeat this calculation with changing the value of $R_{c}$.

Figure 1 plots $p(\cos \theta)$ as dots with the Poissonian errors for the cases of $R_{c}=0,3,6$ and 9 in unit of $h^{-1} \mathrm{Mpc}$ in the four left panels, respectively. The horizontal dotted line in each panel represents the case of no alignment. As one can see, for the case of $R_{c}<3 h^{-1} \mathrm{Mpc}$, the galaxy velocities tend to be anti-aligned with the directions normal to the pancakes. This tendency can be understood as due to the attractive forces exerted among very close neighbors on one another. For the case of $R_{c}=3 h^{-1} \mathrm{Mpc}$ the anti-alignment tendency almost disappears and $p(\cos \theta)$ looks like an uniform distribution having no alignment. When $R_{c} \geq 6 h^{-1} \mathrm{Mpc}$, obvious signals of velocity-pancake alignments appear, which are found to be significant at $99.9 \%$ confidence level.

We find the center of mass (CM) for the given and the two neighbor galaxies and compute the mean value of the distances from CM to the three galaxies. The pancake scale, $L_{p}$, is defined as this mean distance. We then calculate the average of the cosine of the alignment angle, $\langle\cos \theta\rangle$, as a function of $L_{p}$. Figure 2 plots the result as histogram in the upper panel. The horizontal dotted line represents the case of no alignment $\langle\cos \theta\rangle=0.5$. As can be seen, the pancaking effect on the galaxy velocity field exists when the value of $L_{p}$ lies in the range of $5-8 h^{-1} \mathrm{Mpc}$ and gradually dies off at $L_{p} \geq 10 h^{-1} \mathrm{Mpc}$. We refer to this range of $L_{p}=5-8 h^{-1} \mathrm{Mpc}$ where the pancaking effect is significant as the characteristic pancake scales.

Now that we have found a clear signal of the pancaking effect on the galaxy velocity 
field, it will be interesting to test whether the alignment tendency still exists or not when a local pancake is determined using three nearest neighbor field galaxies. Note that if the pancake is determined in this way, then the given galaxy does not necessarily lie on the plane of the pancake.

The results are shown in the four right panels of Fig. 1. Obviously, a very similar tendency of the velocity-pancake alignment is detected for this case, too. It implies that that the signal of velocity-pancake alignment is robust, having a spatial coherence. We also recalculate $\langle\cos \theta\rangle$ as a function of $L_{p}$ for this case, the result of which is plotted as histogram in the lower panel of Fig.2. As can be seen, the characteristic pancake scale becomes slightly larger, $L_{p}=6-10 h^{-1} \mathrm{Mpc}$ for this case.

Using those galaxy-pancake pairs which show the alignment tendency, we investigate the dependence of the pancaking effect on the magnitude of galaxy's velocity $(v)$, the local number density of galaxies $\left(N_{g}\right)$, the pancake's planarity $(\cos \alpha)$, and the galaxy-pancake distance $\left(S_{d}\right)$. Here, the value of $N_{g}$ is obtained by counting the number of all galaxies (both field and cluster) inside a fixed radius of $6 h^{-1} \mathrm{Mpc}$. And, the value of $S_{d}$ represents the separation distance from the given galaxy to the pancake plane that is determined using the three nearest neighbors. The lower panel of Fig. 3 illustrates how $S_{d}$ is defined.

The upper panel of Fig. 3 illustrates the configuration of the three galaxies on the pancake plane and how the pancake planarity is defined. Among the three relative angles made by the three galaxy position vectors from the $\mathrm{CM}$, the angle $\alpha$ is chosen as the one whose cosine has the smallest absolute value. Note that the pancake planarity is lowest if $\cos \alpha \approx 1$, which in fact corresponds to the case of filamentary shape.

We calculate $\langle\cos \theta\rangle$ as a function of $v, N_{g}, \cos \alpha$ and $S_{d}$, the results of which are plotted as dots with errors in the upper left, upper right, lower left, and lower right panels of Fig. 4 , respectively. The errors here are computed as the standard deviation for the case of no correlation: $\sqrt{\left(\left\langle\cos ^{2} \theta\right\rangle-\langle\cos \theta\rangle^{2}\right) / N}$ where $\left\langle\cos ^{2} \theta\right\rangle=1 / 3,\langle\cos \theta\rangle=1 / 2$, and $N$ is the number of galaxies in each bin. As can be seen, the degree of the velocity-pancake alignment increases sharply with $v$ and $N_{g}$, indicating those galaxies with high velocity located near massive pancakes experience stronger pancaking effect.

As can be seen in the lower left panel of Fig.4, the velocity-pancake alignment effect disappears for the case that the pancakes have very low planarity (i.e., $\cos \alpha \sim 1$ ). The lower right panel of Fig. 4 reveals another intriguing result that the strongest signal of the velocity-pancake alignment effect is attained when the distance to the pancake, $S_{d}$, is approximately $2 h^{-1} \mathrm{Mpc}$. Note, however, that compared with the results shown in the other panels the actual signal that attains a maximum at $S_{d} \sim 2 h^{-1} \mathrm{Mpc}$ is much smaller. 
Now, we would like to discuss the difficulty of detecting the pancaking effect in practice. The signal of the velocity-pancake alignment effect is revealed to be very low. Observational detection of it would require very accurate measurement of the positions and the velocities of millions of galaxies and thus may not be achievable from the currently available data. Notwithstanding, by developing a new statistical quantification of the pancaking effect on the galaxy velocity field, it might be possible to observe the effect. Our future project is in this direction and hope to report the result somewhere else in the future.

As final conclusions, we have, for the first time, investigated quantitatively the anisotropy in the galaxy velocity field and found that the galaxy velocities have a tendency to be aligned in the directions normal to the local pancakes of characteristic scales of $5-8 h^{-1} \mathrm{Mpc}$. Our work may provide a deeper insight to the intrinsic properties of the large-scale structure in the universe.

We thank the anonymous referee who helped us improve significantly the original manuscript. The Millennium Run simulation used in this paper was carried out by the Virgo Supercomputing Consortium at the Computing Centre of the Max-Planck Society in Garching. The semi-analytic galaxy catalogue is publicly available at http://www.mpa-garching.mpg.de/galform/agnpaper. J.L. acknowledges stimulating discussion with E.L. Turner. This work is supported by the research grant No. R01-2005-00010610-0 from the Basic Research Program of the Korea Science and Engineering Foundation. 


\section{REFERENCES}

Bardeen, J.M., Bond, J.R.,Kaiser, N., \& Szalay, A.S. 1986, ApJ, 304, 15

Barnes, J., \& Efstathiou, G. 1987, ApJ, 319, 575

Binggeli, B. 1982, A\&A, 107, 338

Bond, J., R., Kofman, L., \& Pogosyan, D. 1996, Nature, 380, 603

Colless et al. 2001, MNRAS, 328, 1039

Croton, D. J., Springel, V., White, S. D. M., De Lucia, G., Frenk, C. S., Gao, L., Jenkins, A., Kauffmann, G., Navarro, J. F., \& Yoshida, N. 2006, MNRAS, 367, 864

El-Ad, H., \& Piran, T. 1997, ApJ, 491, 421

Flin, P., \& Godlowski, W. 1986, MNRAS, 222, 525

Garrido, J. L., Battaner, E., Sanchez-Saavedra, M. L., \& Florido, E. 1993, A\&A, 271, 84

Hoyle, F., \& Vogeley, M. S. 2002, ApJ, 566, 641

Lee, J., \& Pen, U. L. 2001, ApJ, 555, 106

Lee, J., \& Pen, U. L. 2002, ApJ, 567, L111

Lee, J. 2004, ApJ, 614, L1

Navarro, J.F., Abadi, M.G., \& Steinmetz, M. 2004, ApJ, 613, L41

Mo, H. J., Yang, X., van den Bosch, F. C., \& Katz, N. 2005, MNRAS, 363, 1155

Pauls, J. L., \& Melott, A. L. 1995, MNRAS, 274, 99

Shandarin, S. F., Melott, A. L., McDavitt, K., Pauls, J. L., \& Tinker, J. 1995, Phys. Rev. Lett., 75,7

Spergel, D. N. et al. 2003, ApJ, 148, 175

Springel, V. et al. 2005, Nature, 435, 629

Trujillo, I., Carretero, C., \& Patiri, S. 2006, ApJ, 640, L111

Valinia, A., Shapiro, P. R., Martel, H., \& Vishniac, E. T. 1997, ApJ, 479, 46

Zel'dovich, Ya. B. 1970, A\&A, 5, 84 
This preprint was prepared with the AAS LATEX macros v5.2. 


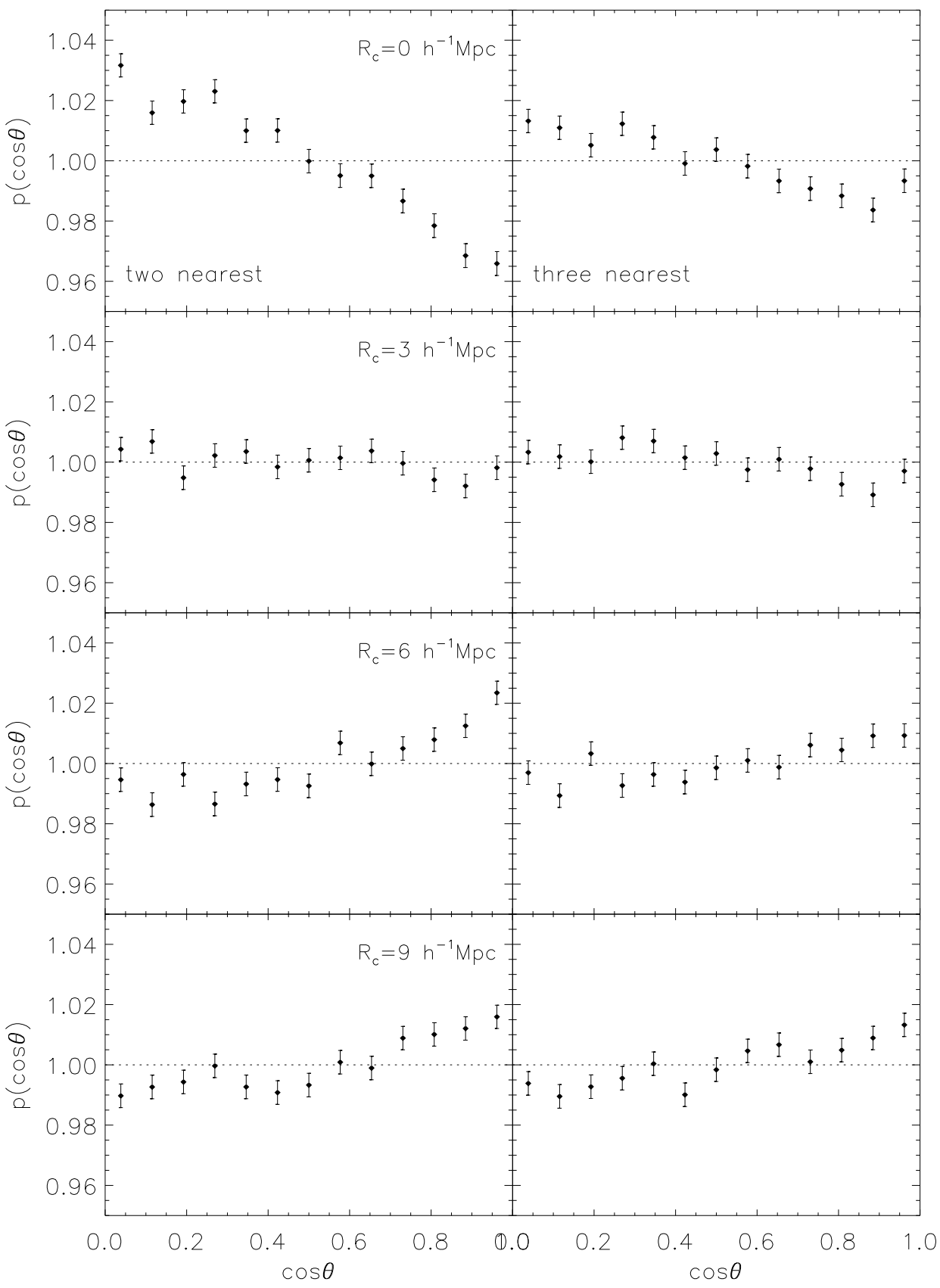

Fig. 1.- Probability density distributions of the cosines of the relative angles between the velocities of the field galaxies and the directions normal to the local pancakes for the cases of the distance threshold: $R_{c}=0,3,6,9 h^{-1} \mathrm{Mpc}$ (from the upper to the lower panels). The data is taken from the Millennium run semi-analytic galaxy catalog (Springel et al 2005). The left four panels correspond to the case that a pancake is determined as a plane encompassing a given galaxy and its two nearest neighbors, while the right four panels correspond to the case that a pancake for a given galaxy determined as a plane encompassing its three nearest neighbors. The errors are Poissonian and the horizontal dotted line corresponds to the case of no alignment in each panel. 

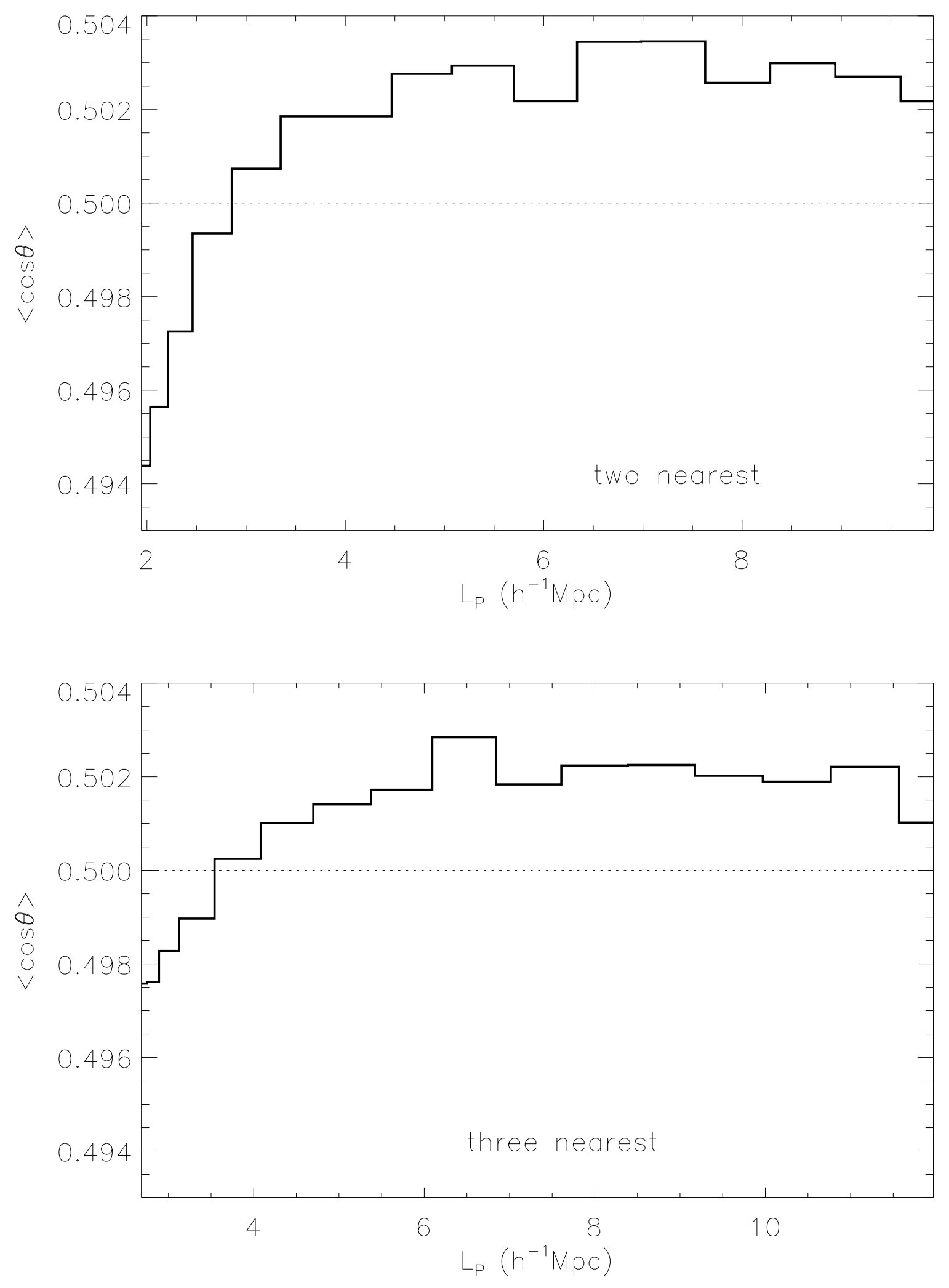

Fig. 2.- Average of the cosines of the alignment angles as a function of the linear size of the pancake, $L_{p}$. The upper panel corresponds to the case that a pancake is determined as a plane encompassing the given galaxy and the two neighbor galaxies, while the lower 


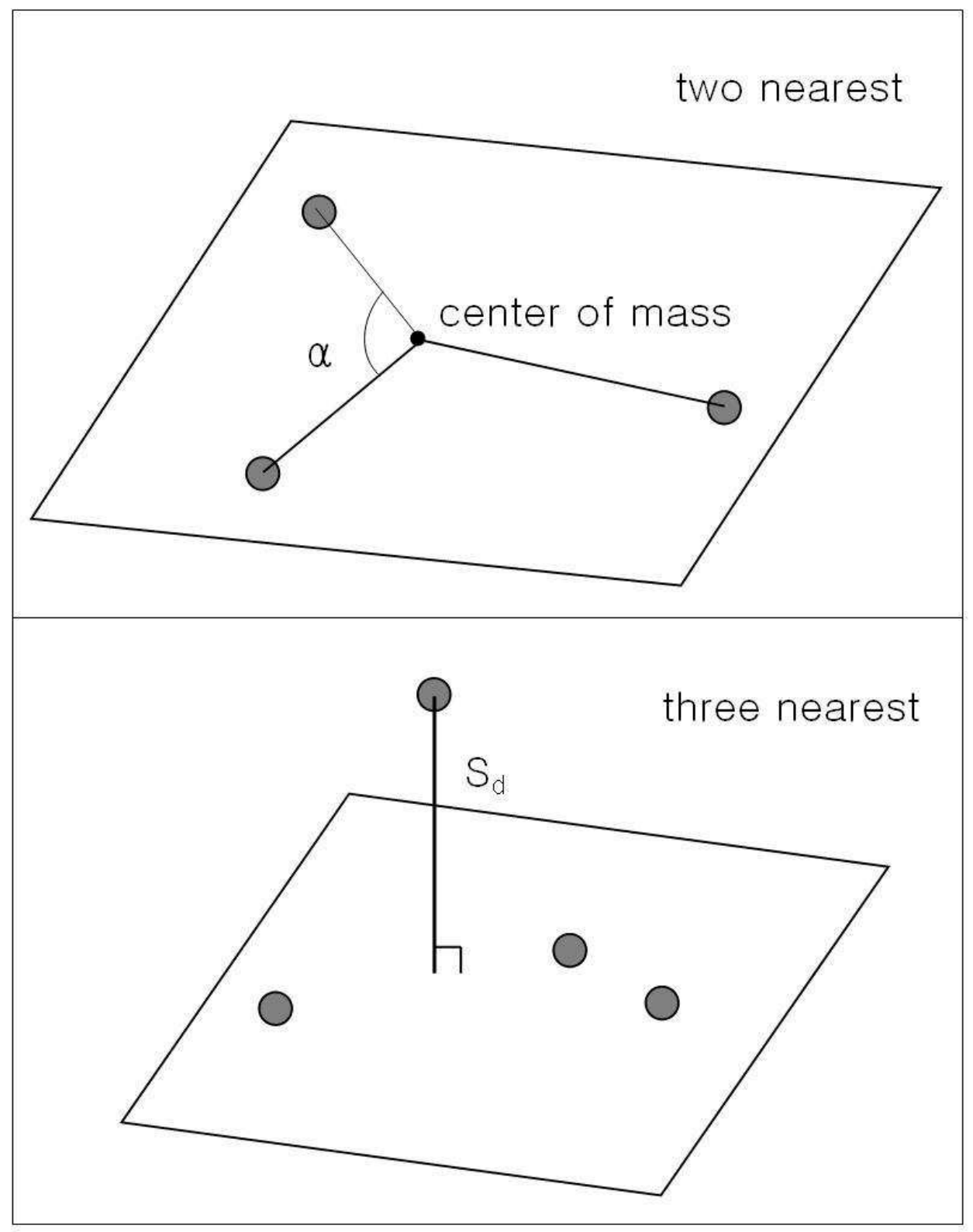

Fig. 3.- The upper panel illustrates the configuration of the relative angle, $\alpha$, when the local plane is defined as a plane encompassing the given galaxy and its two neighbor galaxies. The lower panel panel depicts the value of $S_{d}$ which is the distance between the given galaxy and the pancake plane enclosing three neighbor galaxies. 

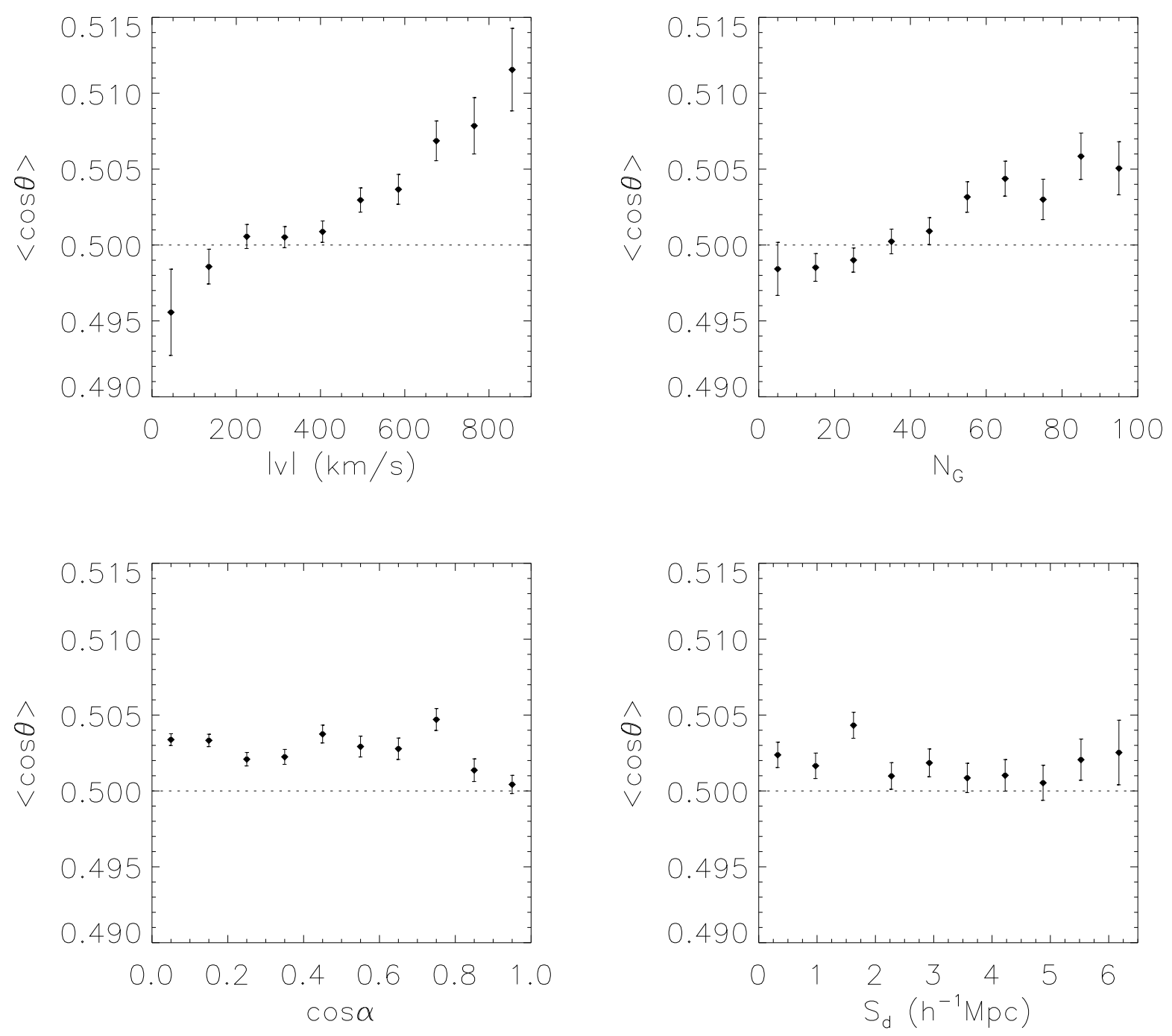

Fig. 4.- Average of the cosines of the alignment angles as a function of the galaxy's velocity magnitude (upper left), number density of galaxies within the distance of $6 h^{-1} \mathrm{Mpc}$ (upper right), the largest cosines of angles between the neighbor galaxies (lower left), and the distance between the galaxy and the pancake plane (lower right). The horizontal dotted line in each panel corresponds to the case of no alignment. 